\title{
The effect of microbubbles on developed turbulence
}

\author{
Irene M. Mazzitelli and Detlef Lohse \\ Department of Applied Physics and J. M. Burgers Centre for Fluid Dynamics, University of Twente, \\ P.O. Box 217, 7500 AE Enschede, The Netherlands
}

Federico Toschi

Istituto per le Applicazioni del Calcolo, CNR, Viale del Policlinico 137, 00161 Rome, Italy and INFM, Unità di Tor Vergata, Via della Ricerca Scientifica 1, 00133 Rome, Italy

(Received 27 August 2002; accepted 22 October 2002; published 4 December 2002)

\begin{abstract}
The motion and the action of microbubbles in homogeneous and isotropic turbulence are investigated through (three-dimensional) direct numerical simulations of the Navier-Stokes equations and applying the Lagrangian approach to track the bubble trajectories. The forces acting on the bubbles are added mass, drag, lift, and gravity. The bubbles are found to accumulate in vortices, preferably on the side with downward velocity. This effect, mainly caused by the lift force, leads to a reduced average bubble rise velocity. Once the reaction of the bubbles on the carrier flow is embodied using a point-force approximation, an attenuation of the turbulence on large scales and an extra forcing on small scales is found. (C) 2003 American Institute of Physics.
\end{abstract}

[DOI: $10.1063 / 1.1528619]$

A fundamental understanding of turbulent two-phase flow is essential for various applications in chemical industry, reactor technology, or the food industry, to name only a few fields. Recent work has either focused on the forces acting on single particles or bubbles ${ }^{1-5}$ or on collective effects, such as dispersion or local concentration evolution. ${ }^{6}$ Among the latter one can distinguish between work focusing on passive particles or bubbles, i.e., without momentum transfer to the flow, ${ }^{7-11}$ and work taking into account the back reaction of the particles and bubbles on the flow (twoway coupling). ${ }^{12-18}$

In turbulent flow initially uniformly distributed bubbles cluster in regions of low pressure and high vorticity. ${ }^{8,19}$ Also experiments with isolated vortices ${ }^{20}$ confirm that bubbles are trapped by them and, moreover, tend to collect on their downflow side. That bubbles can modify the turbulence spectrum has been demonstrated ${ }^{21}$ for void fraction above $1 \%$ where bubbles modify the inertial range scaling, with the energy spectrum power law $-5 / 3$ being gradually substituted by a $-8 / 3$ slope. Also numerical work ${ }^{14,18}$ shows that twoway coupled microbubbles can reduce the turbulent energy in decaying turbulence (depending on the initial bubble distribution) and in turbulent mixing layers under certain conditions.

In this paper we numerically analyze how microbubbles move in and act on three-dimensional developed turbulence. The aim is to demonstrate that above mentioned energy reduction process is rather general and to highlight the role of the lift force therein, which had been neglected in Refs. 14 and 18. Indeed, as already shown on theoretical grounds, ${ }^{5,22}$ the lift on microbubbles cannot be neglected.

Forces on the bubble. The microbubbles are assumed to be spherical with fixed radius $a$, which is small compared to the scales on which the flow varies, and their density is considered to be negligible as compared to the fluid density. The bubble motion can then be modeled by the equation ${ }^{11,16,17,23}$

$$
\begin{aligned}
\frac{d \mathbf{v}}{d t}= & 3 \frac{D \mathbf{u}}{D t}+\frac{1}{\tau_{b}}(\mathbf{u}(\mathbf{y}(t), t)-\mathbf{v}(t))-2 \mathbf{g} \\
& -(\mathbf{v}(t)-\mathbf{u}(\mathbf{y}(t), t)) \times \boldsymbol{\omega}(\mathbf{y}(t), t),
\end{aligned}
$$

where $\mathbf{u}(\mathbf{x}, t)$ is the turbulent velocity field, $\mathbf{y}(t)$ is the bubble position, $\mathbf{v}(\mathbf{x}, t)$ the bubble velocity, $\boldsymbol{\omega}(\mathbf{x}, t)=\nabla \times \mathbf{u}(\mathbf{x}, t)$ the fluid vorticity, $\mathbf{g}$ the gravity, and $\tau_{b}$ a time scale connected with the rise velocity of a bubble in still fluid $v_{T}=2 g \tau_{b}$. The material derivative $(D / D t)$ of the fluid velocity is evaluated at the bubble position.

The four terms on the right hand side of Eq. (1) represent the fluid acceleration plus added mass effects, the drag force, the buoyancy, and the lift force, respectively. The expression of the added mass ${ }^{24,25}$ is appropriate even for viscous nonuniform flows, independently of the Reynolds number. ${ }^{2-4,26}$ The drag force is derived by assuming that no impurities are placed at the bubble-water interface so that the fluid can slip on it, and that the bubble Reynolds number $\operatorname{Re}_{b}=2 a \mid \mathbf{u}$ $-\mathbf{v} \mid / \nu$ is smaller than 1 . Under these constraints the bubble response time $\tau_{b}$ is connected to the radius $a$ and the fluid kinematic viscosity $\nu$ according to $1 / \tau_{b}=6 \nu / a^{2} .{ }^{27,28}$ For intermediate values of the Reynolds number $\left(1<\operatorname{Re}_{b}<60\right)$ a different relation has been found by means of numerical simulations: ${ }^{4} 1 / \tau_{b}=6 \nu / a^{2}\left(1+0.16 \mathrm{Re}_{b}^{1 / 2}\right)$. The expression of the lift force ${ }^{29}$ is reasonable for bubbles with $\operatorname{Re}_{b} \sim O(1)$, like the ones analyzed throughout this work. More accurate values, yet close to the one employed here, can be found in Refs. 1 and 5. Note that, however, other expressions that suppose a stronger lift action and a different dependence on the local vorticity have also been proposed. ${ }^{30}$

Simulation of the flow. Direct numerical simulation is employed in order to simulate the velocity field $\mathbf{u}(\mathbf{x}, t)$. The 
flow satisfies the three-dimensional, incompressible NavierStokes equations,

$$
\frac{\partial \mathbf{u}}{\partial t}+\mathbf{u} \cdot \nabla \mathbf{u}=-\frac{1}{\rho} \nabla p+\nu \Delta \mathbf{u}+\mathbf{f}_{L}+\mathbf{f}_{b} .
$$

Rather than solving Eq. (2), we consider the temporal evolution of the vector potential $\mathbf{b}$, defined according to $\mathbf{u}(\mathbf{x}, t)$ $=\nabla \times \mathbf{b}$. The equations for $\mathbf{b}$ are solved by means of the pseudospectral method. They are advanced in time by second order Adams-Bashforth scheme. The computational domain is a cube of side $L_{0}=2 \pi$ subjected to periodic boundary conditions. The grid consists of $128^{3}$ points. A statistically stationary state is achieved and sustained by forcing the turbulence at large scales. The forcing on the kth mode of the velocity field is

$$
\mathbf{f}_{L}(\mathbf{k}, t)=\varepsilon \frac{\mathbf{u}(\mathbf{k}, t)}{\sum_{\mathbf{k} \in K_{i n}}\left|\mathbf{u}_{i}(\mathbf{k}, t)\right|^{2}}, \quad \mathbf{k} \in K_{\text {in }}
$$

and $\quad \mathbf{f}_{L}(\mathbf{k}, t)=0 \quad$ otherwise. Here $K_{\text {in }}=\left\{\mathbf{k} \mid L_{0} / 2 \pi \mathbf{k}\right.$ $= \pm(-1,2,2), \pm(2,-1,-1)+$ Permutations $\}.{ }^{31}$ Without energy input by bubbles and in the statistically stationary case, the total energy input rate $\left\langle\Sigma_{\mathbf{k} \in K_{i n}} \mathbf{u}^{*}(\mathbf{k}, t) \mathbf{f}_{L}(\mathbf{k}, t)\right\rangle$ then equals $\varepsilon$, which is the viscous dissipation rate. In the present simulation we fix the viscous dissipation $\varepsilon=1.0$ and the kinematic viscosity $\nu=0.007$ from which the Kolmogorov length, time and velocity scales are, respectively, $\eta$ $=\left(\nu^{3} / \varepsilon\right)^{1 / 4}=0.024, \quad \tau_{k}=(\nu / \varepsilon)^{1 / 2}=0.084$ and $v_{k}=(\varepsilon \nu)^{1 / 4}$ $=0.29$, and the Taylor-Reynolds number is $\operatorname{Re}_{\lambda}=62$.

The bubble back reaction on the flow is taken into account by using the point-force approximation. ${ }^{15}$ The forcing is ${ }^{9,16}$

$$
\mathbf{f}_{b}(\mathbf{x}, t)=\sum_{i=1}^{N_{b}}\left(\frac{D}{D t} \mathbf{u}\left(\mathbf{y}_{i}(t), t\right)-\mathbf{g}\right) \mathcal{V}_{b} \delta\left(\mathbf{x}-\mathbf{y}_{i}(t)\right) .
$$

$\mathbf{y}_{i}(t)$ is the Lagrangian position of the $i$ th bubble at time $t, \mathcal{V}_{b}$ is its volume and $N_{b}$ is the total number of bubbles. This form of the back reaction force can be viewed as the force of a "hole" made in the fluid by the bubble, and consequently the missing water cannot transfer momentum, resulting in the additional forcing (4). ${ }^{16}$ The code is completely parallelized, both for the fluid and for the bubbles motion, by using MPI (message passing interface) and simulations are run on SGI Origin2000. A typical run on 16 processors over three large eddy turnover times with $N_{b}=144000$ bubbles takes around 8 hours of CPU time. In a companion paper our employed method is extensively described and justified.

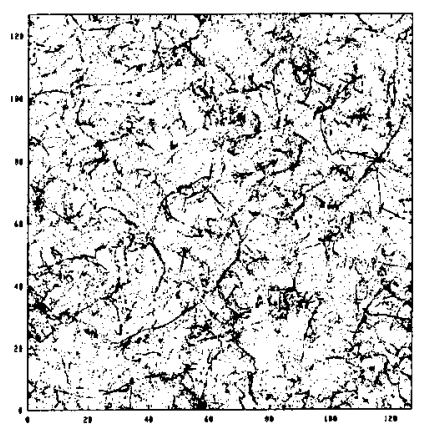

(a)

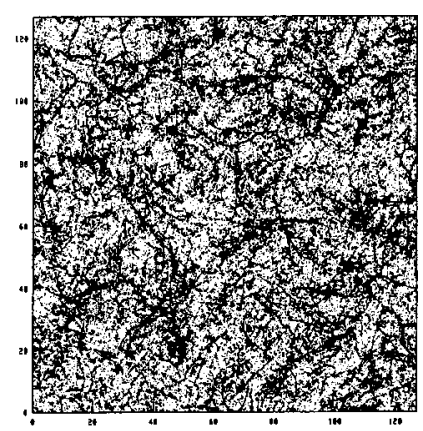

(b)
FIG. 1. Projection of the bubbles distribution for $\tau_{b}=\tau_{k}$ [case (a), left] and $\tau_{b}=\tau_{k} / 3$ [case (b), right]. Bubble clustering is less pronounced in the latter case.

Bubbles local distribution in coherent structures: The role of the lift force. When a bubble is rising in water, it locally transfers momentum mainly upwards, in the direction opposite to gravity [see Eq. (4)]. Therefore it is important to identify the structures in which bubbles are preferentially accumulating, as the back reaction may lead to an enhancement or to a suppression of the velocity fluctuations in these regions.

Previous investigations show that particles moving in a turbulent flow fall down faster than in still fluid whereas bubbles rise slower than in nonturbulent flow. ${ }^{7,9,10}$ This effect is attributed to the phenomenon of "preferential sweeping" 7 of particles and bubbles in downwards fluid velocity regions. In this section we study and quantify this trend for bubbles, identifying the lift force as the main origin of this effect. To this purpose we set, for the time being, $\mathbf{f}_{b}=0$.

In the following, all quantities regarding the disperse phase are estimated by averaging over a sample of $N_{b}$ $=144000$ bubbles, whose motion is evolved according to Eq. (1). Since the bubbles are not actively coupled to the flow $\left(\mathbf{f}_{b}=0\right)$, the void fraction is not a relevant parameter in this context. Simulations are carried out in four distinct bubble regimes. The value of $v_{T}$ is fixed in all cases and equal to $v_{k}$, whereas $\tau_{b}$ is changed: (a) $\tau_{b}=\tau_{k}$, (b) $\tau_{b}$ $=\tau_{k} / 3$, (c) $\tau_{b}=\tau_{k} / 6$, and (d) $\tau_{b}=\tau_{k} / 10$; e.g., for $\varepsilon$ $=0.5 \mathrm{~m}^{2} / \mathrm{s}^{3}$ and $\nu=10^{-6} \mathrm{~m}^{2} / \mathrm{s}$ case (a) corresponds to bubbles of radius $a=\sqrt{6 \nu \tau_{b}}=0.1 \mathrm{~mm}$ with $v_{T}=3 \mathrm{~cm} / \mathrm{s}$. The bubbles are released at time $t=0$ at random locations, with initial velocity equal to the fluid velocity at their position and

\begin{tabular}{|c|c|c|c|c|c|c|c|c|}
\hline & $a$ & $b$ & $c$ & $d$ & $a^{\prime}$ & $c^{\prime}$ & $e$ & $f$ \\
\hline$\tau_{b} / \tau_{k}$ & 1 & $1 / 3$ & $1 / 6$ & $1 / 10$ & 1 & $1 / 6$ & 1 & 1 \\
\hline$v_{T} / v_{k}$ & 1 & 1 & 1 & 1 & 1 & 1 & 2 & 4 \\
\hline lift & yes & yes & yes & yes & no & no & yes & yes \\
\hline$\langle\Omega\rangle_{b} /\langle\Omega\rangle$ & 2.3 & 2.12 & 1.86 & 1.63 & 2.7 & 1.87 & 2.2 & 2.2 \\
\hline$\left(\langle v\rangle_{b}-v_{T}\right) / v_{T}$ & -0.72 & -0.53 & -0.35 & -0.21 & -0.45 & -0.21 & -0.67 & -0.62 \\
\hline$N_{-} / N_{b}$ & 0.58 & 0.56 & 0.54 & 0.53 & 0.51 & 0.53 & 0.64 & 0.7 \\
\hline
\end{tabular}

TABLE I. Results on the mean enstrophy $\langle\Omega\rangle_{b}$, the relative reduction in bubble rise velocity $\left(\langle v\rangle_{b}-v_{T}\right) / v_{T}$, and the relative number $N_{-} / N_{b}$ of bubbles at positions with downwards velocity for various runs with different bubble time scale $\tau_{b}$ and velocity scale $v_{T}$. 


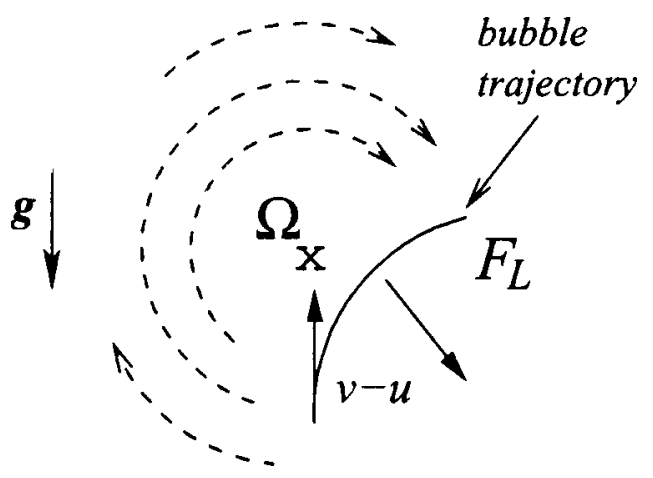

FIG. 2. The lift force acting on a bubble rising in a horizontal vortex pushes it to the side with downward velocity [cf. Eq. (1)].

after a few eddy-turnover times the system reaches a statistically stationary state.

From a 2D projection of the 3D bubbles locations (Fig. $1)$ the clustering (in the vortex tubes) is evident. It can be quantified by measuring the ratio $\langle\Omega\rangle_{b} /\langle\Omega\rangle$ (see Table I), i.e., the time averaged enstrophy at the bubble positions normalized with the average flow enstrophy. This ratio is larger than 1, indicating clustering in high vorticity zones. As in Ref. 9, we find clustering to be most pronounced when the bubble parameters are equal to the flow Kolmogorov scales, $v_{T}=v_{k}$ and $\tau_{b}=\tau_{k}$. Further ratios given in Table I are $N_{-} / N_{b}$, the fraction of bubbles that are sampling downflow regions, and $\left(\langle v\rangle_{b}-v_{T}\right) / v_{T}$, the relative reduction of the bubble rise velocity as compared to its value in still fluid. The values obtained are in qualitative agreement with former investigations. ${ }^{8,9}$ They show that the bubbles preferably accumulate on downflow sides of vortices and therefore the average rise velocity of bubbles is reduced.

To find out the reason for this effect, two parallel simulations, $\left(a^{\prime}\right)$ and $\left(c^{\prime}\right)$, were carried out, in which the lift force is switched off (Table I). Now the trapping effect of the bubbles in the high enstrophy regions is even more pronounced, but the symmetry breaking between upwards and downwards flow sampling bubbles is much less and correspondingly the reduction in rise velocity is also less. We conclude that it is mainly the lift force which makes the bubbles drift towards the side of the vortex with downward velocity. Indeed, this can be directly inferred from Eq. (1). ${ }^{11}$ A sketch of the dynamics is shown in Fig. 2.

The effect can be enhanced in simulations with larger gravity force in Eq. (1), i.e., (e) $2 g \tau_{b}=v_{T}=2 v_{k}$ and (f) $v_{T}$ $=4 v_{k}$ instead of (a) $v_{T}=v_{k}$ (in all cases $\tau_{b}=\tau_{k}$ ). First, the larger gravity force partially counteracts the bubble accumulation in the central zone of the vortices, leading to a smaller ratio $\langle\Omega\rangle_{b} /\langle\Omega\rangle$. Second, because of the larger bubble velocity $v_{T}$, the lift force increases, leading to a larger ratio $N_{-} / N_{b}$ of bubbles on the downward flow side of the vortices. ${ }^{32}$

Bubbles coupling to the carrier flow: Turbulence modulation. We now study "active" bubbles [i.e., $\mathbf{f}_{b}$ as in Eq. (4)] with $\tau_{b}=\tau_{k} / 10$ and terminal velocity $v_{T} \simeq v_{k}$. The reason why we choose this (small) response time is to restrict our analysis to small bubble radii, in order to apply the point force approximation. The ratio $a / \eta=0.8, N_{b}=144000$ and

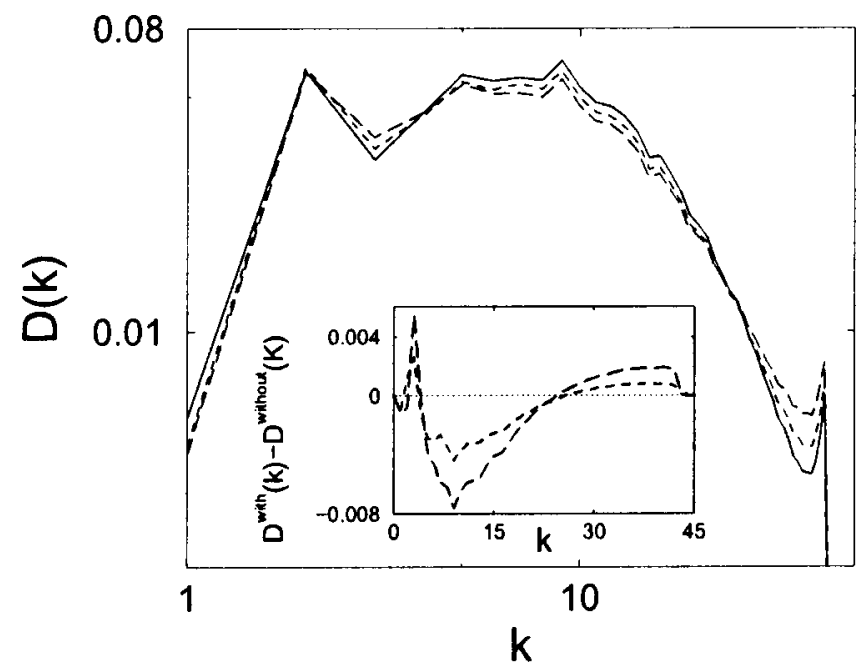

FIG. 3. Fluid dissipative spectra in cases (A) (dashed) and (B) (long dashed) compared to the single phase spectrum (solid). The inset shows the difference between the dissipative spectra of the single phase flow $D^{\text {without }}(k)$ and case (A) (dashed) and (B) (long dashed).

the void fraction is $\alpha \simeq 1.6 \%$. Bubble-bubble interactions are neglected. This regime corresponds to microbubbles of diameter $d \simeq 150 \mu \mathrm{m}$ in clean water. The bubble response time is kept constant and the rise speed is changed. We focus our attention on the two cases (A) $v_{T}=2 v_{k}$ and (B) $v_{T}$ $=4 v_{k}$.

For two-way coupling clustering in vortices and preferential sweeping still exist, though in a more modest form as compared to the one-way coupling case, as already found in Ref. 14. Our main finding for the two-way coupling case is that the bubble action on the flow is selective in wavenumber: in Fig. 3 the dissipative spectra $D(k)=2 \nu k^{2} E(k)$ are compared to the spectrum for single phase flow. The spectrum with two-way coupling is reduced with respect to the single-phase turbulence at large scales and enhanced at small scales. Consequently, bubbles act as a sink of energy at large scale, whereas they force the flow at small scale.

In the competition between large scale and small scale effect we find that, for the Taylor-Reynolds number treated here $\left(\operatorname{Re}_{\lambda}=62\right.$ which is hardly modified through the two-way coupling), dissipation overwhelms the forcing: the overall effect is to reduce the turbulent dissipation rate. We find this result remarkable as in pseudoturbulence (flow forcing exclusively through rising bubbles) the rising bubbles of course induce extra dissipation. In Fig. 4 the total energy dissipation is plotted as a function of the ratio $v_{T} / v_{k}$. Note that, without bubbles, $\varepsilon=1$. The curve has a minimum at $v_{T} / v_{k} \simeq 3-4$, corresponding to $v_{T}=0.9-1.2$. The physical explanation of our numerical finding is as follows: microbubbles, accumulated in downflow regions, locally transfer momentum upwards (see Fig. 2). Consequently, they reduce the intensity of the vertical velocity fluctuations and therefore also of the turbulent kinetic energy. The strongest effect is achieved when the bubble velocity scale $v_{T}$ is of the order of the rms velocity fluctuation $u_{0} \simeq 1.2$. Then the bubble interaction with the energy containing large scale structures is most efficient and the dissipation reaches its minimum. An energy 


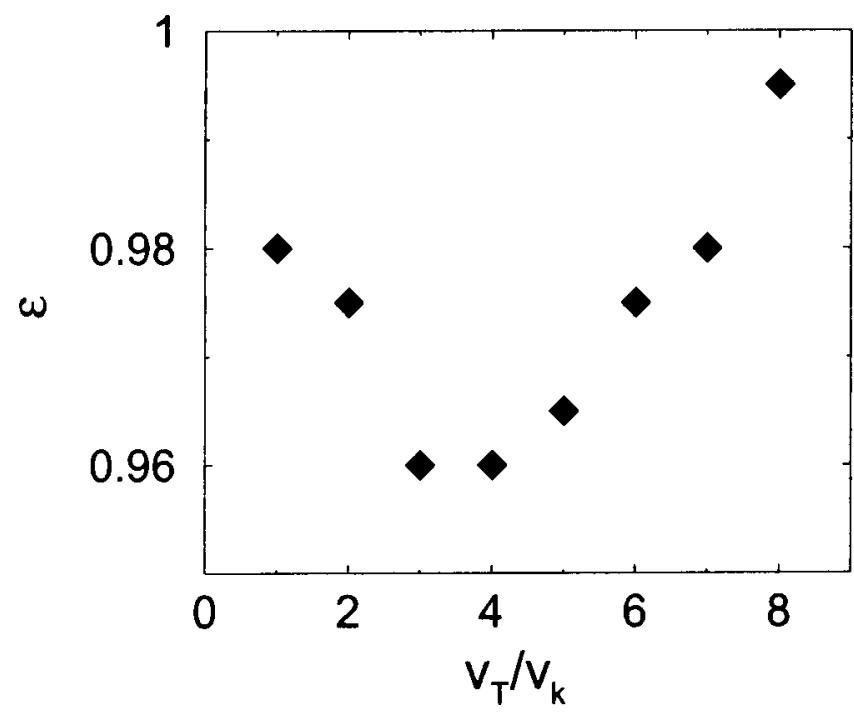

FIG. 4. Fluid viscous dissipation, $\varepsilon$, as a function of the ratio $v_{T} / v_{k}$.

reduction on large scales and an energy enhancement on small scales may lead to a modification of the spectral scaling exponent. Owing to the suggested mechanism the energy spectrum slope will be less steep than in one phase turbulence. Moreover, it will depend on the number of bubbles and will therefore be non-universal. ${ }^{33}$

\section{ACKNOWLEDGMENTS}

The authors thank E. Climent, A. Prosperetti, and L. van Wijngaarden for very helpful discussions and TIM for the CPU time. The work is part of the research program of the Stichting voor Fundamental Onderzoek der Materie (FOM), which is financially supported by the Nederlandse Organisatie voor Wetenschappelijk Onderzoek (NWO).

${ }^{1}$ J. Magnaudet and I. Eames, "The motion of high-Reynolds number bubbles in inhomogeneous flows," Annu. Rev. Fluid Mech. 32, 659 (2000)

${ }^{2}$ M. Rivero, J. Magnaudet, and J. Fabre, "New results on the forces exerted on a spherical body by an accelerated flow," C. R. Acad. Sci., Ser. II 312, 1499 (1991).

${ }^{3}$ E. Chang and M. Maxey, "Unsteady flow about a sphere at low to moderate Reynolds number. Part 2: Accelerated motion," J. Fluid Mech. 303, 133 (1995).

${ }^{4}$ J. Magnaudet, M. Rivero, and J. Fabre, "Accelerated flows past a rigid sphere or a spherical bubble. Part 1: Steady straining flow," J. Fluid Mech. 284, 97 (1995).

${ }^{5}$ D. Legendre and J. Magnaudet, "The lift force on a spherical bubble in a viscous linear shear flow," J. Fluid Mech. 368, 81 (1998).

${ }^{6}$ C. T. Crowe, T. Troutt, and J. N. Chung, "Numerical models for two-phase turbulent flows," Annu. Rev. Fluid Mech. 28, 11 (1996).

${ }^{7}$ L. Wang and M. Maxey, "Settling velocity and concentration distribution of heavy particles in homogeneous isotropic turbulence," J. Fluid Mech. 256, 27 (1993).

${ }^{8} \mathrm{~L}$. Wang and M. Maxey, "The motion of microbubbles in a forced isotropic and homogeneous turbulence," Appl. Sci. Res. 51, 291 (1993).
${ }^{9}$ M. Maxey, E. Chang, and L. Wang, "Simulation of interactions between microbubbles and turbulent flows," Appl. Mech. Rev. 46(6), S70 (1994).

${ }^{10} \mathrm{C}$. Yang and U. Lei, "The role of the turbulent scales in the settling of heavy particles in homogeneous isotropic turbulence," J. Fluid Mech. 371, 179 (1998)

${ }^{11}$ P. D. M. Spelt and A. Biesheuvel, "On the motion of gas bubbles in homogeneous isotropic flow,” J. Fluid Mech. 336, 221 (1997).

${ }^{12}$ K. D. Squires and J. K. Eaton, "Particle response and turbulence modification in isotropic turbulence," Phys. Fluids A 2, 1191 (1990).

${ }^{13} \mathrm{~S}$. Elghobashi and G. Truesdell, "On the two-way interaction between homogeneous turbulence and dispersed solid particles. I: Turbulence modification," Phys. Fluids A 5, 1790 (1993).

${ }^{14}$ O. A. Druzhinin and S. Elghobashi, "Direct numerical simulations of bubble-laden turbulent flows using the two-fluid formulation," Phys. Fluids 10, 685 (1998).

${ }^{15}$ M. Boivin, O. Simonin, and K. Squires, "Direct numerical simulation of turbulence modulation by particles in isotropic turbulence," J. Fluid Mech. 375, 235 (1998).

${ }^{16} \mathrm{E}$. Climent, "Dispersion de bulles et modification du mouvement de la phase porteuse dans des ecoulements tourbillonnaires," Ph.D. thesis, Inst. Nat. Polytech. Toulouse, 1996.

${ }^{17}$ E. Climent and J. Magnaudet, "Large-scale simulations of bubble-induced convection in a liquid layer," Phys. Rev. Lett. 82, 4827 (1999).

${ }^{18}$ O. A. Druzhinin and S. Elghobashi, "Direct numerical simulation of a three-dimensional spatially developing bubble-laden mixing layer with two-way coupling," J. Fluid Mech. 429, 23 (2001).

${ }^{19} \mathrm{O}$. Cadot, S. Douady, and Y. Couder, "Characterization of the lowpressure filaments in a 3-dimensional turbulent shear-flow," Phys. Fluids 7, 630 (1995).

${ }^{20}$ G. Sridhar and J. Katz, "Effect of entrained bubbles on the structure of vortex rings," J. Fluid Mech. 397, 171 (1999).

${ }^{21} \mathrm{M}$. Lance and J. Bataille, "Turbulence in the liquid phase of a uniform bubbly air-water flow," J. Fluid Mech. 222, 95 (1991).

${ }^{22}$ K. J. Sene, J. C. R. Hunt, and N. H. Thomas, "The role of coherent structures in bubble transport by turbulent shear flows," J. Fluid Mech. 259, 219 (1994).

${ }^{23}$ N. H. Thomas, T. R. Auton, K. Sene, and J. C. R. Hunt, "Entrapment and transport of bubbles in plunging water," in Gas Transfer at Water Surfaces, edited by W. Brutsaert and G. H. Jiska (Reidel, Dordrecht, 1983), pp. $255-268$.

${ }^{24} \mathrm{G}$. Taylor, "The forces on a body placed in a curved of converging stream of fluid," Proc. R. Soc. London, Ser. A 120, 260 (1928).

${ }^{25}$ G. K. Batchelor, An Introduction to Fluid Dynamics (Cambridge University Press, Cambridge, 1967).

${ }^{26}$ T. R. Auton, J. Hunt, and M. Prud'Homme, "The force exerted on a body in inviscid unsteady non-uniform rotating flow," J. Fluid Mech. 197, 241 (1988).

${ }^{27}$ J. Hadamard, "Mouvement permanent lent d'une sphere liquide et visqueuse dans un liquide visqueux," C. R. Acad. Sci. Paris 152, 1735 (1911).

${ }^{28} \mathrm{~W}$. Rybczynski, "On the translating motion of a fluid sphere in a viscous medium," Bull. Acad. Sci. Cracovie 40, 40 (1911).

${ }^{29}$ T. Auton, "The lift force on a spherical body in a rotational flow," J. Fluid Mech. 183, 199 (1987).

${ }^{30}$ G. Sridhar and J. Katz, "Drag and lift forces on microscopic bubbles entrained by a vortex," Phys. Fluids 7, 389 (1995).

${ }^{31}$ S. Grossmann and D. Lohse, "Intermittency in the Navier-Stokes dynamics," Z. Phys. B: Condens. Matter 89, 11 (1992).

${ }^{32}$ The relative reduction of the bubble rise velocity $\left(\langle v\rangle_{b}-v_{T}\right) / v_{T}$ however is smaller due to the increase of $v_{T}$.

${ }^{33}$ Note that this result does not contradict the experimental finding reported in Ref. 21. The regimes analyzed are different: in our case $\mathrm{Re}_{b} \sim O(1)$, so the flow around the bubble is laminar. In Ref. $21 \operatorname{Re}_{b} \gg 1$, leading to a turbulent wake behind the bubble, which according to Ref. 21 leads to the $-8 / 3$ scaling exponent found in that paper. 